\title{
CARCINOMA ESOFÁGICO DE CÉLULAS ESCAMOSAS - RELATO DE CASO
}

\author{
ESOPHAGEAL CARCINOMA OF SCALY CELLS- CASE REPORT
}

\author{
Luana Silva e Silva dos Anjos ${ }^{1}$, Gboado Emmanuella Richenel Hermine \\ Akowanou $^{2}$, Nickolas Cezar Isecke ${ }^{3}$, Luiz Guilherme Pereira Medeiros ${ }^{4}$, \\ Raymundo do Espírito Santo Pedreira 5 .
}

\begin{abstract}
RESUMO
A neoplasia de esôfago é relativamente rara e marcada por alta mortalidade. Com maior incidência em homens acima de 50 anos, está fortemente associada ao tabagismo e etilismo. Seu diagnóstico na maioria das vezes é tardio devido aos sintomas iniciais que são vagos podendo sugerir outas patologias do trato digestivo. Existem 20 tipos histológicos porem dois são mais importantes: o tipo epidermóide e o adenocarcinoma. O local mais acometido é o terço-médio do esôfago associado à doença do refluxo e esôfago de Barret. O prognóstico varia conforme o tipo histológico e o estágio do tumor. É necessária uma abordagem minuciosa dos pacientes da população de risco, mesmo que apresentem queixas esofágicas incipientes, para esta doença. Em razão disso, a descrição epidemiológica científica, fatores como idade, história familiar e fatores extrínsecos como álcool e tabagismo devem ser de alerta para pesquisa desta neoplasia para um diagnóstico e tratamento o mais precoce possível e aumento da sobrevida desses pacientes.
\end{abstract}

Citação: Anjos LSS, Akowanou GERH, Isecke NK, Medeiros LGP, Pedreira RES (2019) Carcinoma esofágico de células escamosas - relato de caso. Revista de Patologia do Tocantins, 6(1): 31-32.

Instituição: ${ }^{1}$ Acadêmica de Medicina, Universidade Federal do Tocantins, Palmas, Brasil; ${ }^{2}$ Acadêmica de Medicina, Universidade Federal do Tocantins, Palmas, Brasil; ${ }^{3}$ Acadêmico de Medicina, Universidade Federal do Tocantins, Palmas, Brasil; ${ }^{4}$ Acadêmico de Medicina, Universidade Federal do Tocantins, Palmas, Brasil; ${ }^{5}$ Médico Radiologista, Instituto Tocantinense Presidente Antônio Carlos, Porto Nacional, Brasil, Universidade Federal do Tocantins, Palmas, Brasil; Secretaria Estadual de Saúde, Palmas, Brasil e Hospital Regional de Porto Nacional, Porto Nacional, Brasil.

Autor correspondente: Luana Silva e Silva dos Anjos; sssluh@gmail.com

Editor: Guedes V. R. Medicina, Universidade Federal do Tocantins, Brasil.

Publicado: 06 de maio de 2019.

Direitos Autorais: (c) 2019 Anjos et al. Este é um artigo de acesso aberto que permite o uso, a distribuição e a reprodução sem restrições em qualquer meio, desde que o autor original e a fonte sejam creditados.

Conflito de interesses: os autores declararam que não existem conflitos de interesses.

\begin{abstract}
Esophageal neoplasia is relatively rare, with a high mortality. With greater incidence in men over 50 years old, associated with smoking and alcohol consumption. Its diagnosis is most often late due to initial symptoms that are vague and may suggest other pathologies of the digestive tract. There are 20 histological types, but two are more important: the carcinoma of scaly cells and adenocarcinoma. The most affected place is the middle third of the esophagus associated with reflux disease and Barrett's esophagus. The prognosis varies according to the histological type and tumor stage. A thorough approach is required for patients in the at-risk population, even if they have incipient esophageal complaints, for this disease. Therefore, the scientific epidemiological description, the factors like age, family history and extrinsic factors such as alcohol and tobacco should be alert to research of this neoplasm for a diagnosis and treatment as early as possible and increase survival of these patients.

Keywords: Esophageal Neoplasms; Risk factors; early diagnosis.
\end{abstract}


INTRODUÇÃO

O câncer de esôfago é a 6ạ causa de morte no Brasil e a $8^{\text {a }}$ no mundo ${ }^{1}$. Estima-se que somente no ano de 2018 ocorreram 10.790, sendo 8.240 homens e 2.550 mulheres no Brasil com 7.930 mortes (6.203 homens e 1.727 mulheres) ${ }^{2}$. O carcinoma de células escamosas, também chamado de carcinoma epidermóide, tem alta letalidade e é a neoplasia maligna mais comum do esôfago ( $96 \%$ dos casos), seguida do adenocarcinoma, de ocorrência crescente ${ }^{2}$.

Por se tratar de um câncer de prognóstico ruim, dada à alta letalidade e ao diagnóstico tardio, as taxas de mortalidade aproximam-se das taxas de incidência, com ocorrência mais frequente em homens com mais de 50 anos e estreita relação com o tabagismo e alcoolismo. Outros fatores que predisponentes incluem: toxinas fúngicas, deficiências nutricionais, líquidos quentes, carcinógenos químicos, exposição ocupacional e o papiloma vírus humano (HPV 16,18 e 59) que têm um papel de destaque na gênese desses tumores 3,4

A localização mais frequente do câncer de esôfago é o terço médio devido à elevada ocorrência da Doença do Refluxo Gastresofágico (DGRE) crônico e o Esôfago de Barret. O carcinoma de células escamosas pode acometer qualquer parte, mas, especialmente, no terço médio e o inferior ${ }^{4}$.

fator prognóstico mais importante é o estadiamento baseado no sistema TNM; sendo assim os pacientes em que o tumor não ultrapassa a submucosa, a sobrevida pode chegar a $70 \%$ enquanto a sobrevivência é de $30 \%$ a $50 \%$ nos pacientes nos quais há invasão da muscular própria e adventícia ${ }^{4}$. 0 presente relato revela a importância do diagnóstico apropriado do carcinoma escamosa do esôfago no âmbito de terapia precoce e diminuição da morbimortalidade.

\section{RELATO DO CASO}

I.D.S., 71 anos, masculino, negro, casado, lavrador, etilista e tabagista crônico, queixando-se de queimação retroesternal, disfagia progressiva para sólidos e líquidos, vômitos e perda de $21 \mathrm{~kg}$ nos últimos dois meses. O paciente foi submetido à endoscopia digestiva alta que evidenciou lesão vegetante com margens ulceradas, friável e com áreas de necrose. A lesão tinha efeito expansivo que reduzia $90 \%$ da amplitude da luz do esôfago médio.

Diagnóstico tomográfico revelou espessamento circunferencial do esôfago que se estendia do terço médio até a cárdia, promovendo importante estreitamento da luz esofágica com presença de linfonodomegalias locorregionais nas cadeias para-esofágica, para-aórtica e paratraqueal.

À biopsia incisional por método endoscópio com análise histopatológica foi possível evidenciar células atípicas constituindo pequenos blocos irregulares e brotamentos, compatíveis com carcinoma escamoso bem diferenciado.

\section{DISCUSSÃO}

Geralmente o diagnóstico do câncer do esôfago é baseado nos sintomas, embora possa ocorrer acidentalmente em pessoas assintomáticas. Os principais fatores de risco (tabagismo e alcoolismo) agem de forma sinérgica, principalmente no tipo epidermóide, aumentando o risco em 5 10 vezes comparado a não fumantes.

Mutações no gene p53 induzem a carcinogênese mais cedo no tipo epidermóide do que no tipo adenocarcinóide ${ }^{4}$. A expansão tumoral produz disfagia progressiva de alimentos sólidos para líquidos. A odinofagia, desconforto retroesternal, sensação de corpo estranho no esôfago proximal, dor epigástrica, anorexia, náuseas, perda sanguínea e emagrecimento sem causa aparente são outros sintomas a serem avaliados nos paciente ${ }^{5,6}$. $\quad 0$ aparecimento de linfonodos endurecidos, palpáveis na fossa supraclavicular (nódulo de Troisier-Virchow) e na axila (nódulo de Ireland), geralmente significa doença metastática. Essa disseminação pode ocorrer por contiguidade, via linfática, hematogênica ou intramural.

Em razão do curso insidioso e dos sintomas inicialmente inespecíficos, a maioria dos tumores esofágicos já estão avançados à época do diagnóstico, tornando o tratamento mais difícil e o prognóstico reservado, na maioria dos $\operatorname{casos}^{6}$, salientando a importância de um exame minucioso em pacientes que apresentem os fatores cartesianos dessa patologia, especialmente quando achados em sinergia, a saber: etilismo e tabagismo ${ }^{4,5,6}$.

\section{REFERÊNCIAS}

1. Domper Arnal M. J., Ferrández Arenas, Lanas Arbeloa: Esophageal cancer: Risk factors, screening and endoscopic treatment in Western and Eastern countries. World Journal Gastroenterol [Internet]. 2015 Jul 14; 21(26): 7933-7943. Disponível em: https://www.wjgnet.com/1007-9327/full/v21/i26/7933.htm

2. Instituto Nacional de Câncer (INCA); [2018]. Tipos de cânce: Câncer de esôfago [atualizado em 2018]. Disponível em: http://www2.inca.gov.br/wps/wcm/conne

ct/tiposdecancer/site/home/esofago/definicao

3. Mota M. Orlando, Curado Maria, Oliveira José C., Martins Edesio, Cardoso Daniela M. Risk factors for esophageal cancer in a lowincidence area of Brazil. Sao Paulo Medical Journal [Internet]. 31 de Maio de 2012; 131(1): 27-34. Disponível em: http://www.scielo.br/pdf/spmj/v131n1/1516-3180-spmj-131-0127.pdf

4. Oliveira-Borges E. C., Silva Adriana F., Graças Amanda M., Melo Fabiano S. F., Barcelos Adle A., Myiata S. Câncer de esôfago: uma revisão. Revista da Universidade Vale do Rio Verde [Internet]. 14 de Julho de 2015; 13(1): 773-790. Disponível em: file://C:/Users/Adm/Downloads/Dialnet-OCancerDeEsofago5168604\%20(1).pdf

5. Monteiro Nonato M. L., Araújo Daniel F., Soares Eric B., Vieira José P. F., Santos Marcos R. M., Júnior Pedro P. L., Delgado Tatiana S. J. Esophageal Cancer: Profile of Clinical Manifestations, histology, location and metastatic behavior in pacients undergoing cancer treatment at a cancer center in Minas Gerais. Revista Brasileira de Cancrologia [Internet]. 21 de Julho de 2008; 55(1): 27-32. Disponível

em: http://www.inca.gov.br/rbc/n_55/v01/pdf/06_artigo_cancer_de _esofago.pdf

6. American Cancer Society medical Instituto; Esophagus Cancer: Early detection, diagnossis, and staging [updated 2017 June 14]. Available from: https://www.cancer.org/cancer/esophaguscancer/detection-diagnosis-staging/signs-and-symptoms.html 